\title{
La oferta de formación continua para el adulto mayor en Colombia, caso región Caribe
}

\section{The offer of continuing education for the elderly in Colombia, the case of the Caribbean region}

Emperatriz Londoño-Aldana

Universidad de Cartagena - Colombia

ORCID iD: https://orcid.org/0000-0002-9761-0950

emperatriz1521@yahoo.com

María Eugenia Navas-Ríos

Universidad de Cartagena - Colombia

ORCID iD: https://orcid.org/0000-0002-8951-7916

mariaeunavas@yahoo.com

Zilath Romero-González*

Universidad Libre - Colombia

ORCID iD: https://orcid.org/0000-0003-4588-288X

zilathromero@yahoo.com

* Autor a quien debe ser dirigida la correspondencia
Fecha de recepción: 03/01/2020

Fecha de evaluación: 11/05/2020

Fecha de aceptación: 01/06/2020

Cómo citar: Londoño-Aldana, E., Navas-Ríos, M., \& Romero-González, Z. (2020). La oferta de formación continua para el adulto mayor en Colombia, caso región Caribe. Revista Científica Anfibios, 3(1), 78-84. https://doi.org/10.37979/afb.2020v3n1.65

\section{Resumen}

El presente artículo parte del reconocimiento de las personas de la tercera edad como un segmento poblacional con un papel cada vez más relevante en la demanda de educación continua. Sin ignorar el difícil panorama que enfrentan muchos adultos mayores en el país, se asume también que estas personas, contrario a sus homónimos de hace algunas décadas, se han hallado con ciertas condiciones que les permiten mantener una vida más activa. Tanto las ofertas de educación continua on line existentes como las que en el futuro puedan ser adaptadas a sus necesidades específicas, representan, en este contexto, espacios clave a los que pueden acudir en busca de oportunidades para la socialización, el crecimiento personal, y la actualización de conocimientos. Esta es, sin duda, una gran oportunidad para mejorar la calidad de vida de esas personas, al tiempo que se les permite asegurar su vigencia laboral, aunando sus largos años de experiencia a la adquisición de nuevos saberes. Se trata, pues, de una investigación exploratoria que requiere la búsqueda, el análisis y el procesamiento de información secundaria relacionada con prácticas que propicien el envejecimiento activo y productivo. El objetivo es sentar las bases para el desarrollo de un programa de educación continua que se adapte a las necesidades y características propias del adulto mayor en Colombia, específicamente de la región Caribe.

Palabras claves:

Adulto mayor; Educación continua; Prototipo; Conocimientos; Región caribe

\begin{abstract}
This article starts from the recognition of older people as a population segment with an increasingly relevant role in the demand for continuing education. Without ignoring the difficult panorama faced by many older adults in the country, it is also assumed that these people, contrary to their homonyms of some decades ago, have found certain conditions that allow them to maintain a more active life. Both the existing online continuing education offers and those that may be adapted to their specific needs in the future represent, in this context, key spaces to which they can turn in search of opportunities for socialization, personal growth, and the updating of knowledge. This is, without a doubt, a great
\end{abstract}


opportunity to improve the quality of life of these people, while allowing them to ensure their working life, combining their long years of experience with the acquisition of new knowledge. This is, therefore, an exploratory research that requires the search, analysis and processing of secondary information related to practices conducive to active and productive ageing. The aim is to lay the foundations for the development of a continuing education programme that is adapted to the needs and characteristics of older adults in Colombia, specifically in the Caribbean region.

\section{Keywords:}

Older Adult; Continuing education; Prototype; Knowledge; Caribbean Region.

\section{Introducción}

En Colombia, de acuerdo con el Decreto 272 de 2017, se considera "adulto mayor" a toda persona que tenga 60 años o más. La Organización Mundial de la Salud (OMS) hace una clasificación más puntual: en el grupo de "edad avanzada" se encuentran quienes estén entre 60 y 74 años; las personas "viejas o ancianas" son aquellas con edades entre 75 y 90 años, mientras que quienes sobrepasen los 90 años son denominados "grandes viejos" o "grandes longevos" (OMS, s. f.).

De acuerdo con las estimaciones de la OMS (s. f.), "entre 2000 y 2050, la proporción de los habitantes del planeta mayores de 60 años se duplicará, pasando del $11 \%$ al 22\%", representado en unos 2000 millones de personas. Como es de esperarse, Colombia no escapa a esta tendencia. La población de adultos mayores en nuestro país ha crecido vertiginosamente en los últimos años. Según Rodríguez (2016), el número de personas mayores de 60 años creció $153 \%$ entre 1988 y 2016, pasando de 2’358.360 a 5'970.956.

Dicho crecimiento se ha debido, en parte, a la existencia de mejores condiciones de vida en relación con la salud, la educación y la capacidad de pago de esta población, la cual se sustenta gracias a la recepción de salarios fijos, mesadas pensionales, ayudas de familiares y subvenciones del Estado, entre otras fuentes de ingreso. Aunque el panorama no deja de ser desalentador para muchas de esas personas, especialmente por la falta de asistencia social y la necesidad de trabajar en la informalidad para garantizar su propia subsistencia (Fernández, 2018), también es cierto que una buena parte de ellas posee menos obligaciones y responsabilidades (tienen resueltos gastos grandes como hijos, estudio, casa y carro), lo cual les permite invertir en servicios y comodidades a las que antes no tenían acceso, dinamizando de manera importante la economía.

Pertenecer al grupo de personas mayores de 60 años significa experimentar una serie de cambios importantes, tanto biológicos como afectivos y sociales. De acuerdo con Cruz y Pérez (2006), estos últimos representas uno de los factores más decisivos en la calidad de vida de esta población, toda vez que debe enfrentar la necesidad de ajustar los roles y funciones antes desempeñados, especialmente debido a la desvinculación con el mercado laboral. Este tránsito plantea, en la sociedad actual, un serio problema de integración laboral, pues quienes quedan inactivos ven fácilmente deteriorada su salud mental y física.

Esto pone de relieve el hecho de que el trabajo sigue ocupando un rol central en la definición del status y la identidad de las personas, así como en sus posibilidades de integración a la sociedad y, por tanto, su reconocimiento y autoestima. Sin embargo, también hay otras formas de mantenerse activos e integrados, entre las cuales el aprendizaje juega un rol muy importante. De hecho, la formación para adultos es un sector en crecimiento. Hay muchas personas de edad avanzada que se plantean estudiar, ya sea porque lo dejaron para más adelante o porque no les fue posible en el pasado. Así mismo, hay otros adultos que entienden los estudios como una forma de distraerse o de conocer gente.

En respuesta a esta realidad, el presente artículo plantea la necesidad de realizar un sondeo en la situación de los adultos mayores de nuestro país, específicamente en la región Caribe, a fin de elaborar una propuesta capaz de atender a las necesidades de formación de este segmento poblacional. 


\section{Metodología}

El presente artículo plantea la necesidad de realizar una investigación cualitativa, basada en la exploración de la bibliografía existente sobre la situación actual (y proyectada) de los adultos mayores en relación con la educación para toda la vida. Se espera que el análisis de los datos secundarios obtenidos por medio de este método, permita formular soluciones a la problemática de integración social que representa la mayoría de edad para un creciente segmento de la población nacional, especialmente en lo relativo a las oportunidad de socialización y mejora de la calidad de vida que ofrece la formación continua.

\section{Una propuesta de educación continua para el adulto mayor}

La actual población de adultos mayores en Colombia se encuentra integrada por personas que, a diferencia de sus homólogos de décadas anteriores, poseen la vitalidad y la capacidad física para seguir desarrollando roles muy activos en la sociedad. Entre otros indicadores de esta situación, sobresale la disposición de muchos adultos mayores a seguir aprendiendo para mantenerse intelectualmente activos. Es innegable el valor que esto tiene para mejorar calidad de vida de quienes, por cuestión de edad, se han retirado del mercado laboral. No tenerlo en cuenta implica desconocer el derecho de los ciudadanos a la integración social, especialmente en quienes resultan tan vulnerables frente a problemas psicosociales y están tan expuestos a toda forma de violencia.

Abrir el abanico de posibilidades de formación para estas personas, además de las oportunidades de socialización ya expuestas, ofrece incluso la ventaja de mantenerlas en contacto con la realidad del ámbito laboral al cual han pertenecido o, simplemente, poder vincularse a otros espacios. Normalmente, quienes se jubilan saben desempeñarse solamente en labores o funciones que realizaban cuando se encontraban activos, de manera que, si quisieran ser productivos en otras labores, se les presenta una serie de barreras por encontrarse desactualizados. También se encuentran quienes, por el contrario, simplemente desean aprender cosas nuevas y enriquecedoras, que les permitan estar ocupados, crecer y seguir siendo productivos.

Por otra parte, quienes no estudiaron o no lograron trabajar en lo que les gustaba, desean capacitarse en áreas del conocimiento relacionadas con lo que en su momento consideraron era el camino para su vida profesional. Así mismo, están quienes de jóvenes no pudieron ir a la universidad y, una vez retirados, contemplan la oportunidad de estudiar una carrera como una acto de realización personal.

En este contexto, se espera que los resultados de la investigación aporten información que propicie un cambio de mentalidad general en torno a la idea de la desvinculación de este segmento como algo natural y necesario (Palmore \& Maeda, 1985), al tiempo que se despierta el interés de las instituciones educativas por ofertar planes de capacitación para adultos mayores en el marco de la responsabilidad social que les compete, facilitando que se actualicen y sean activos durante muchos años más.

\section{Sistemas de recomendación para el aprendizaje continuo}

La convergencia entre las nuevas tecnologías de la información para el procesamiento de datos y la educación, provee importantes oportunidades para la mejora de la calidad de vida de los ciudadanos mayores. La implementación de tecnologías de la información basadas en macrodatos (big data) ha permitido el acceso a nuevas fuentes de datos que antes eran inaccesibles. Con esto se ha abierto la puerta a grandes innovaciones en el campo de la ciencia y la industria que hoy representan notables conquistas en la mejora de la calidad de vida (Ruiz-Navas \& Miyazaki, 2018).

Como era de esperarse, estos avances han permitido la convergencia entre el big data y la educación. Un ejemplo del resultado de esta integración se puede apreciar en plataformas de educación online como Coursera y Edex, que proveen cursos masivos abiertos (en inglés Massive Open Online Courses o MOOC). Los datos generados en el proceso de aprendizaje 
(en línea o presencial) pueden ser de varios tipos, y van desde el historial de notas, hasta la asistencia a los cursos y las técnicas de enseñanza más apropiadas para grupos específicos de personas.

Esta información se recopila y se procesa en tiempo real, dando a las instituciones la oportunidad de conocer el movimiento de los estudiantes dentro del campus o el nivel de flujo dentro de un ambiente de aprendizaje virtual, todo ello en relación con grupos de personas tan grandes como pueden ser los estudiantes de todo un país o los miembros inscritos en una determinada plataforma de enseñanza.

En otros términos, el uso de tecnologías de big data hace posible mejorar la calidad de la educación y promover el aprendizaje individual de formas impensables hace unos años (Ye, 2019). En el marco del uso de estas tecnologías, los "sistemas de recomendación" son implementados para ayudar en la toma de decisiones a quienes han decidido emprender un proceso de formación. En el caso de los aprendices, estos sistemas les ayudan a seleccionar los cursos o los materiales de estudio más apropiados. En cuanto a las instituciones, les permiten crear currículos personalizados e implementar políticas para la mejora del rendimiento académico de los aprendices.

Los sistemas de recomendación se han usado también para mejorar la calidad de vida de los adultos mayores, ya sea permitiéndoles seleccionar y preparar menús personalizados (Ribeiro et al., 2017) o facilitando el diseño de programas de salud capaces de responder a las exigencias propias de su edad (Cheung et al., 2019). De ahí que no sea descabellado pensar en la posibilidad de poner estas tecnologías al servicio de la formación continua de adultos mayores en Colombia. Dicha labor incluiría la recolección de información sobre intereses y necesidades específicas de esta población, así como el diseño y la implementación de programas de enseñanza accesibles, acordes con lo que estas personas buscan al momento de vincularse a un proceso formativo.

Quien quiera llevar a cabo esta empresa debe tener en cuenta que, tal como lo afirman
Casado (2002) y Cabanes (2005), en los adultos mayores tiende a incrementarse el miedo a lo desconocido gracias a las limitaciones propias de la edad, como son la disminución de la agudeza visual y auditiva, la destreza manual, etc. Sin embargo, también puede contar con la capacidad de los seres humanos para adaptarse a las circunstancias que les toca vivir. En este sentido, Cruz-Díaz \& Román-García (2015) coinciden en afirmar que, si bien es cierto que la relación de la generación mediática (los adultos mayores de hoy) que creció bajo el protagonismo de la televisión como medio de comunicación más destacado ha sido más o menos difícil, la generación digital de los seniors del futuro, acostumbrados desde su juventud o edad adulta al uso de las nuevas tecnologías, puede hallar en ellas el entorno ideal para seguir formándose cuando hayan llegado a la edad de retirarse.

Afortunadamente, en varios países de Europa y América se están haciendo importantes avances en este sentido. En Francia, las instituciones educativas, así como las asociaciones que ofrecen actividades para las personas mayores, incluyen ofertas de entrenamiento formal (seminarios, cursos) e informal (clubes, comunidades, redes sociales) (Boutrand, 2009) que tienen como referente común la Universidad de la Tercera Edad de Tolouse, fundada en 1973 con la idea de brindar programas de enseñanza, investigación y servicio a la colectividad.

En el caso de España, a inicios del siglo XXI destaca la Universidad de las Palmas de Gran Canaria con el programa Peritia et Doctrina (experiencia y doctrina - lo que saben y pueden aprender), el cual constituye una interesante alternativa en cuanto a medidas de protección social y atención a la tercera edad del nuevo milenio. En México, el Instituto Nacional de las Personas Adultas Mayores (Inapam) propicia que estas personas puedan llevar a cabo actividades productivas, a través de programas de capacitación para el Trabajo y Ocupación del Tiempo Libre. Del mismo modo, busca asegurar la instrumentación de programas para promover empleos y trabajos remunerados, así como actividades lucrativas o voluntarias para las personas adultas mayores, conforme a su oficio, habilidad o profesión, sin más restricción que su limitación física o mental. 
En Cartagena de Indias, Lambis \& Roca (2016) llevaron a cabo un estudio sobre el "potencial productivo de los profesionales mayores de 60 años de la ciudad". Uno de sus resultados más significativos tiene que ver con la refutación de la idea generalizada de que, en la medida en que aumenta el rango de edad entre los adultos mayores, se va disminuyendo la disposición a seguir llevando a cabo actividades productivas. Contrario a lo que se cree, su comportamiento no es lineal descendente.
En el rango de edad de 60 a 65 años, el 83\% de las personas encuestadas durante el estudio están de acuerdo y totalmente de acuerdo en seguir siendo productivas. Cuando se pasa al rango de 66 a 72 años, este porcentaje disminuye considerablemente, pasando al $37.5 \%$. Sin embargo, al subir al rango de edad de 73 a 80 años, la disposición a seguir realizando actividades productivas repunta hasta un $50 \%$, el cual llega al $66.6 \%$ en personas de más de 80 años.

Tabla 1. Disposición a realizar actividades productivas entre personas mayores de 60 años.

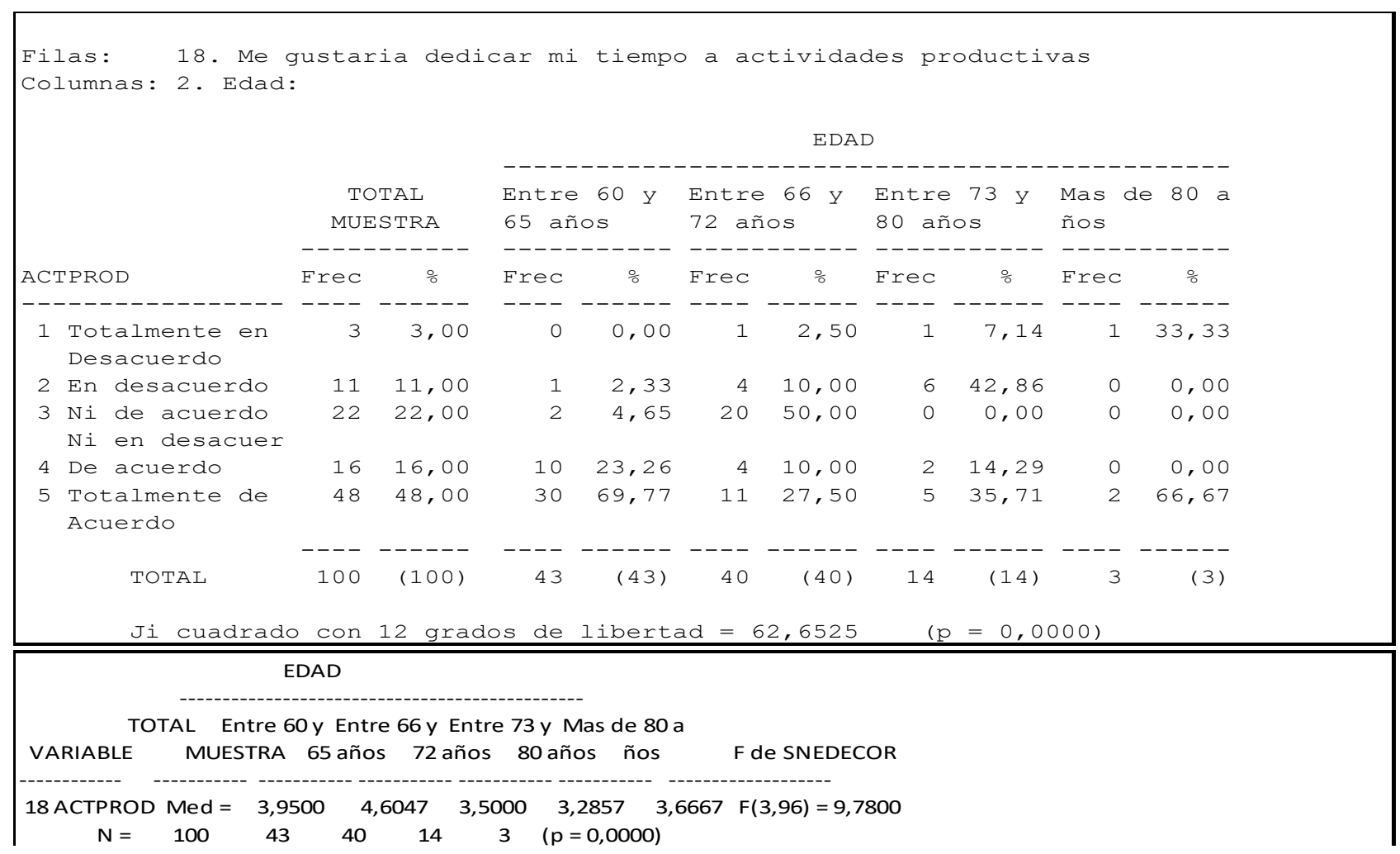

Nota: Las diferencias anotadas en las afirmaciones por rango de edades frente a la opción de "seguir siendo productivos" resultan significativas, dado que al aplicar las pruebas $\mathrm{Ji}^{2}$ y $\mathrm{F}$ de Snedecor se obtuvo un valor $\mathrm{p}$ inferior al 5\% (p=0\%). Fuente: Lambis \& Roca (2016).

Fuente: Elaboración de los autores

Frente a este escenario, se hace cada vez más evidente la necesidad de estructurar una oferta de capacitación continua, adecuada a las necesidades físicas, biológicas, psicológicas, sociales y laborales de todas aquellas personas pertenecientes a la tercera edad que conservan buena disposición para seguir realizando actividades productivas. También se convierte en un reto para los docentes, de acuerdo con Fernández Guerrero, M., \& Gonzalez Ferro, V. (2017) el profesor, como persona y como profesional de la enseñanza, debe estar en sintonía con los nuevos escenarios, debe estar preparado para enseñar, el arte de transformar y crecer, posibilitando la participación de este grupo poblacional en actividades académicas de acuerdo con sus intereses particulares. Lograr este objetivo, daría a las universidades y otras instituciones educativas la oportunidad de atender integralmente los compromisos sociales ligados a su misión y cumplir con su responsabilidad social (Navas Rios, M., \& Romero-González, Z. (2016). 


\section{Conclusiones}

A pesar de los prejuicios y las formas de discriminación que existen en torno a la edad madura, este periodo de la vida puede resultar perfecto para aprender y disfrutar de espacios de integración social, especialmente porque la tendencia es que, a mayor edad, las personas dispongan de mayor tiempo libre. De hecho, está claro que muchos de los adultos mayores siguen teniendo deseos de aprender cosas nuevas y realizar actividades que les permitan mantenerse activos. En otros países es común hallar espacios de formación tendientes a permitir que estas personas puedan seguir avanzando, lo cual demanda una buena disposición de las instituciones públicas y privadas para responder a las necesidades, motivaciones, expectativas y experiencias que posee este gran segmento de la población.
La investigación aquí esbozada corresponde precisamente al deseo de dar respuesta a dicha necesidad, en el contex to propio de la región Caribe colombiana. Para ello, se insiste en la importancia de conocer a fondo la situación de los adultos mayores, de modo que puedan desarrollarse estrategias de formación capaces de atender a sus realidades y expectativas. En tal sentido, será de suma importancia aprovechar al máximo el rol que actualmente juegan las nuevas tecnologías de la información. No es un secreto que el uso de éstas por parte de las personas de tercera edad representa un problema internacional de competitividad empresarial. Por esa razón, no basta con utilizarlas para diseñar los programas de formación, sino que ellas mismas deben constituir un tema de estudio entre las distintas opciones de aprendizaje que se ofrezcan a los adultos mayores.

\section{Referencias}

Boutrand, M. (2009): Seniors et Cité. République Française: Conseil Économique, Social et Environnemental. https://www.lecese.fr/sites/default/files/pdf/Avis/2009/2009_08_\%20monique_boutrand.pdf

Cabanes, L. (2005). Tercera edad, necesidad de educación en el adulto mayor. Revista Digital. Lecturas de Educación Física y Deportes [Revista en Línea]. http//www.monografias.com/trabajos23/tercera-edad-educación/tercera-edadeducacion.shtml

Casado, J. \& Gregorio, P. (2002). Función mental y envejecimiento. Editores Médicos.

Cheung, K., Durusu, D., Sui, X. \& de Vries, H. (2019). How recommender systems could support and enhance computer-tailored digital health programs: A scoping review. Digital Health, 5. https://doi.org/10.1177/2055207618824727

Cruz, A. \& Pérez, L. (2006). Envejecer ayudando, envejecer aprendiendo. Adultos mayores en Uruguay: actores del voluntariado y del servicio cívico. Montevideo: Instituto de Comunicación y Desarrollo (ICD).

Fernández Guerrero, M., \& Gonzalez Ferro, V. (2017). Enseñar, el arte de transformar y crecer. Saber, Ciencia Y Libertad, 12(2), 167-174. https://doi.org/10.18041/2382-3240/saber.2017v12n2.1584

Fernández, C. F. (22 de mayo de 2018). El desalentador panorama del adulto mayor en Colombia. Revista Portafolio. https://www.portafolio.co/economia/panorama-del-adulto-mayor-en-colombia-2018-517356

Lambis L. \& Roca A. (2016). Potencial productivo de los profesionales mayores de 60 años de la ciudad de Cartagena. Tesis de grado para optar por el título de Administrador de Empresas. Universidad de Cartagena. Colombia

Navas Rios, M., \& Romero-González, Z. (2016). Responsabilidad social universitaria. Saber, Ciencia Y Libertad, 11(1), 187-196. https://doi.org/10.18041/2382-3240/saber.2016v11n1.503

Organización Mundial de la Salud. (s. f.). Envejecimiento y ciclo de vida. https://www.who.int/ageing/es/

Palmore, E. \& Maeda, D. (1985). The honorable elders revisited: a cross-cultural analysis of aging in Japan. Durham, North Carolina: Duke University Press. 
Pavón, F., Cruz-Díaz \& Román-García, S. (2015). Del uso de las tecnologías de la comunicación a las destrezas en competencia mediática en las personas mayores. Redes, 12, 87-111.

Ribeiro, F., Santos, G., Rebelo, M. F. \& Silva, R. (2017). Integrated management systems: Trends for portugal in the 2025 horizon. Procedia Manufacturing, 13, 1191-1198.

Rodríguez, C. (20 de septiembre de 2016). El mercado del envejecimiento. Revista Portafolio. https://www.portafolio.co/tendencias/el-mercado-del-envejecimiento-500464

Ruiz-Navas, S., \& Miyazaki, K. (2018). Developing a framework to track knowledge convergence in'big data'. International Journal of Technology Intelligence and Planning, 12(2), 121-151. https://doi.org/10.1504/IJTIP.2018.096101

Ye, C. (2018). The Research Development of Big Data in Education: A Bibliometric Analysis Based on Citespace, Seventh International Conference of Educational Innovation through Technology (EITT), Auckland, New Zealand, 2018, pp. 116-122. https://doi.org/10.1109/EITT.2018.00031. 